\title{
Compact strange stars with a medium dependence in gluons at finite temperature
}

\author{
M. Bagchi ${ }^{1}$, S. Ray ${ }^{2}$, M. Dey ${ }^{1, \star}$, and J. Dey ${ }^{1}$ \\ 1 Dept. of Physics, Presidency College, 86/1, College Street, Kolkata 700073, India \\ e-mail: mnj2003@vsnl.net; kamal1@vsnl.com \\ 2 Inter University Centre for Astronomy and Astrophysics (IUCAA), Post Bag \# 4, Ganeshkhind, Pune 411007, India \\ e-mail: sray@iucaa.ernet.in
}

Received 30 June 2005 / Accepted 19 December 2005

\section{ABSTRACT}

\begin{abstract}
Aims. The possible existence of strange stars in the universe will help in the understanding of various properties of quantum chromodynamics, like asymptotic freedom and chiral symmetry restoration, which is otherwise very difficult to prove in laboratory experiments.

Methods. Strange star properties were calculated using large color approximation with built-in chiral symmetry restoration. A relativistic Hartree Fock calculation was performed using the Richardson potential as an interquark interaction. This potential has the asymptotic freedom and a confinement-deconfinement mechanism built into it and the present calculation employs an application of this potential with modified two scale parameters $\Lambda$ and $\Lambda^{\prime}$, to find a new set of equations of state for strange quark matter. The linear confinement string tension from lattice calculations is $350 \mathrm{MeV}$ and the Coulomb -like part has the parameter $100 \mathrm{MeV}$ from deep inelastic scattering experiments. We also consider the effect of temperature, on gluon mass in a simple way, in addition to the usual density dependence,

Results. We obtained a set of new equation of states for strange quark matter and also found that the transition temperature from hadronic matter to strange matter is at $80 \mathrm{MeV}$, close to the $100 \mathrm{MeV}$ estimated in literature.

Conclusions. Formation of strange stars may be the only signal for formation of quark-gluon plasma with asymptotic freedom and chiral symmetry restoration and this may be observable through many processes - such as for example through delayed $\gamma$ ray afterglow.
\end{abstract}

Key words. equation of state - dense matter - stars: interiors

\section{Introduction}

Two decades have passed since the existence of strange matter and strange stars was proposed by Witten (1984), and it is still difficult to prove or disprove the existence of a state of strange quark matter in its purest form. Besides the mass radius relation, efforts have been made to find other signatures for the existence of quark stars. The existence of absorption bands at $0.35 \mathrm{keV}$ with harmonics at $0.7,1.05,1.4$ etc., in 1E 1207-5209 was interpreted in Sinha et al. (2003) as evidence for strange stars. It was then observed that six stars show harmonic band emission at energies corresponding to the same compressional modes at which the absorption bands were invoked (Ray et al. 2004). The SPI spectrometer on the INTEGRAL satellite detected a bright $511 \mathrm{keV}$ line in the $\gamma$-ray spectrum from the bulge of the galaxy with a spherically symmetric distribution (Knodleseder et al. 2003). This has stimulated research to the fundamental physics that describes cosmological dark matter. Several authors (Boehm et al. 2004; Oaknin \& Zhitnitsky 2005) have suggested that the line comes

\footnotetext{
* CSIR Emeritus Scientist
}

from positron annihilation, possibly in strange stars made of antiquarks. The implication of cosmic separation of phases (Witten 1984) is that strange stars (SS) can exist since the early universe and can themselves explain dark matter. A crucial condition for the survival of these stars, given by Alcock \& Olinto (1989), is a large value of surface tension $\sim(178 \mathrm{MeV})^{3}$ which can be obtained from strange star equation of state (EOS) (Bagchi et al. 2005). In his recent review, Weber (2005) argued that the constraints on mass radius relations from observational data support that the compact objects SAX J1808.4-3658, 4U 1728-34, RX J1856.5-3754 and Her X-1 are strange stars rather than neutron stars and on the basis of its low value of temperature, PSR 0205+6449 may be considered as a strange star. He also discussed the possibility of explaining some astrophysical phenomena like X-ray bursts, gamma-ray bursts and soft gamma repeaters with a strange star model. The most controversial is the isolated compact star RX J1856.5-3754, which has a featureless thermal spectrum, and its X-ray data fits quite well with a blackbody distribution. With a distance estimate of $120 \mathrm{pc}$, Drake et al. (2002) gave the radiation radius of the star to be $4.12 \pm 0.68 \mathrm{~km}$. This indicates that the stellar radius is $4 \mathrm{~km}$ to $8 \mathrm{~km}$. Later, with a better estimation of the distance 
of $175 \mathrm{pc}$, the estimated radiation radius $R^{\infty} \simeq 8 \mathrm{~km}$. However, there are controversies regarding the surface temperature of this star. With an estimated surface temperature $\left(T^{\infty}\right)$ of $63.5 \mathrm{eV}$, $R^{\infty} \simeq 4.4 \mathrm{~km}$, and with $T^{\infty} \simeq 32 \mathrm{eV}, R^{\infty} \simeq 8 \mathrm{~km}$ (Burwitz et al. 2003).

There is evidence, as recently discussed by Bombaci et al. (2004) - for the existence of compact strange and neutron stars with larger radius and a shift from the latter to the former can account for delayed gamma ray bursts.

PSR $1913+16$ is the first pulsar to be discovered in a double neutron star system is, also known as the Hulse Taylor pulsar (1975). It is estimated to have a mass of $1.441 M_{\odot}$ and the recently discovered pulsar (Lyne et al. 2004) PSR J07373039A, forming a binary pulsar system, has a mass of $1.337 M_{\odot}$. Thus the mass of pulsars may lie near these values, the observed variation of periodicity of the pulsars indicates the variation in their masses. Although it may be a stringent lower-limit constraint for neutron stars masses, which are bound mainly by gravitational forces, the same does not hold for compact objects, which are self bound, like a system of quark matter. Theoretically there is no physical reason to have a lower limit for the mass of strange stars, where strong interactions play an important role in stellar binding. The same EOS will allow even a very small quark nugget.

In the literature, there are several EOSs for strange quark matter, starting from the Bag model (Alcock et al. 1986; Haensel et al. 1986; Kettner et al. 1995) to the recent models like the mean field model with interacting quarks (Dey et al. 1998), the strange star EOS model in the perturbative quantum chromodynamics (QCD) approach (Fraga et al. 2001), those of the chiral chromodielectric model (Malheiro et al. 2003) or Dyson-Schwinger model (Blaschke et al. 1999), etc. In our present work, we will consider only the relativistic mean field model by Dey et al. (1998).

In the relativistic Hartree-Fock calculation of a strange quark matter EOS to form a compact star (Dey et al. 1998), a phenomenological inter-quark interaction, namely the Richardson potential, was used. Strange stars are more compact than conventional neutron stars and fit into the Bodmer (1971) - Witten (1984) hypothesis for the existence of strange quark matter. They were able to produce a very compact stellar structure with a mass of about $1.44 M_{\odot}$ and radius of about $7 \mathrm{~km}$. The original Richardson (1979) potential was designed to obtain the mass spectrum of heavy mesons (Charmonium and Upsilon). It includes two features of the qq force, the asymptotic freedom (AF) and confinement, however, with the same scale, $\Lambda$.

The $\Lambda$ parameter needed was $400 \mathrm{MeV}$. It was further applied to light meson spectroscopy and baryon properties with the same value of $\Lambda$ (Crater \& van Alstine 1984; Dey et al. 1998). Although both the confinement and AF scales are chosen to be the same in the Richardson potential, it is the confinement, not the AF part, that plays the more important role in determining the static properties of hadrons. However, when the AF part is important (as in strange star property calculations), $\Lambda$ would be much smaller, around $100 \mathrm{MeV}$, as it is the scale for AF, obtained from perturbative QCD. Indeed, for self bound high density strange quark matter, Dey et al. (1998) found that $\Lambda$ needed to be $\sim 100 \mathrm{MeV}$. In the present paper, proper scales are chosen for both the parameters, as determined from a calculation by Bagchi et al. (2004) by fitting the masses and recently determined magnetic moments of $\Omega^{-}$and $\Delta^{++}$.

In Sect. 2 we describe the basic formalism of the model as given in Dey et al. (1998) and the calculations incorporating finite temperature effects. In Sect. 3 we present a fine tuning of the Dey et al. (1998) model, with a modified version of the Richardson potential, and also incorporate a temperature dependence of gluon mass. In Sect. 4 we show the results and the plots, and draw conclusions and summarize in Sect. 5.

\section{The model}

There are no free parameters in QCD with which one can systematically build up a perturbation series. So 't Hooft 1974 suggested use of the inverse of the number of colors - which is the key quantum number in QCD - as an expansion parameter. In this case a baryonic system like stars can be explored in relativistic tree level calculations with a potential that may be obtained from the meson sector phenomenology (Witten 1979). In this connection the Richardson potential is a very good choice for interquark interactions.

The original Richardson potential is given by:

$V_{i j}=\frac{12 \pi}{27} \frac{1}{\ln \left(1+\left(\boldsymbol{k}_{i}-\boldsymbol{k}_{j}\right)^{2} / \Lambda^{2}\right)} \frac{1}{\left(\boldsymbol{k}_{i}-\boldsymbol{k}_{j}\right)^{2}}$.

At high density (high Fermi momentum) quarks are deconfined as the bare potential is screened. Confinement is softened and the AF part takes over. This bare potential in a medium will be screened due to gluon propagation and $\left(\boldsymbol{k}_{i}-\boldsymbol{k}_{j}\right)^{2}$ of Eq. (1) is replaced by $\left[\left(\boldsymbol{k}_{i}-\boldsymbol{k}_{j}\right)^{2}+D^{-2}\right]$. The inverse screening length, $D^{-1}$, which can be identified with the gluon mass $m_{\mathrm{g}}$, to the lowest order is:

$\left(D^{-1}\right)^{2}=\frac{2 \alpha_{0}}{\pi} \sum_{i=u, d, s,} k_{i}^{f} \sqrt{\left(k_{i}^{f}\right)^{2}+m_{i}^{2}}$

where $k_{i}^{f}$, the Fermi momentum of the ith quark is obtained from the corresponding number density:

$k_{i}^{f}=\left(n_{i} \pi^{2}\right)^{1 / 3}$

and $\alpha_{0}$ is the perturbative quark gluon coupling.

In this model, chiral symmetry restoration at high density is incorporated by assuming that the quark masses are density dependent:

$M_{i}=m_{i}+M_{Q} \operatorname{sech}\left(\frac{n_{\mathrm{B}}}{N n_{0}}\right), \quad i=\mathrm{u}, \mathrm{d}, \mathrm{s}$

where $n_{\mathrm{B}}=\left(n_{\mathrm{u}}+n_{\mathrm{d}}+n_{\mathrm{s}}\right) / 3$ is the baryon number density; $n_{0}=0.17 \mathrm{fm}^{-3}$ is the normal nuclear matter density; $n_{\mathrm{u}}, n_{\mathrm{d}}$, $n_{\mathrm{s}}$ are the number densities of $\mathrm{u}, \mathrm{d}$ and s quarks respectively and $N$ is a parameter. The current quark masses $\left(m_{i}\right)$ are taken as: $m_{\mathrm{u}}=4 \mathrm{MeV}, m_{\mathrm{d}}=7 \mathrm{MeV}, m_{\mathrm{s}}=150 \mathrm{MeV}$. It is ensured that in strange matter, the chemical potentials of the quarks satisfy $\beta$ equilibrium and charge neutrality conditions. The parameters $M_{Q}$ and $N$ are adjusted in such a way that the 
minimum value of $E / A$ for $\mathrm{u}, \mathrm{d}$, s quark matter is less than that of $\mathrm{Fe}^{56}$, so that $\mathrm{u}, \mathrm{d}, \mathrm{s}$ quark matter can constitute stable stars. The minimum value of $E / A$ is obtained at the star surface where the pressure is zero. The surface is sharp since strong interaction dictates the deconfinement point. An electron cloud forms above this sharp surface - since they are not held by strong interaction. However, the minimum value of $E / A$ for u$\mathrm{d}$ quark matter is greater than that of $\mathrm{Fe}^{56}$ so that $\mathrm{Fe}^{56}$ remains the most stable element in the non-strange world. With the obtained EOSs, they solved the hydrostatic equilibrium equations (Tolman-Oppenheimer-Volkov equation) to get the structure of the stars.

Finite temperature $T$ was incorporated in the system through the Fermi function (Ray et al. 2000b):

$$
F M(k, T)=\frac{1}{\mathrm{e}^{\left(\epsilon-\epsilon_{F}\right) / T}+1}
$$

with the flavour dependent single particle energy

$\epsilon_{i}=\sqrt{k^{2}+M_{i}(\rho)^{2}}+U_{i}(k)$.

$I=\frac{g}{2 \pi^{2}} \int_{0}^{\infty} \phi(\epsilon) k^{2} F M(k, T) \mathrm{d} k$

where $g$ is the spin-colour degeneracy, equal to 6 and $I$ is the number density for $\phi(\epsilon)=1$ and energy density for $\phi(\epsilon)=\epsilon$.

The system is highly degenerate even at very high $T$ which is around $70 \mathrm{MeV}$ since the chemical potential is very high, of the order of several hundred $\mathrm{MeV}$. The entropy was calculated as follows:

$$
\begin{aligned}
S(T)= & -\frac{3}{\pi^{2}} \int_{0}^{\infty} k^{2}[F M(k, T) \ln (F M(k, T)) \\
& +(1-F M(k, T)) \ln (1-F M(k, T))] \mathrm{d} k
\end{aligned}
$$

The pressure was calculated from the free energy

$f=\epsilon-T s$

as follows:

$P=\sum_{i} \rho_{i} \frac{\partial f_{i}}{\partial \rho_{i}}-f_{i}$

Ray et al. (2000b), found that stable star structure cannot be obtained with a temperature greater than $70 \mathrm{MeV}$. But here the effect of temperature on gluon mass was not considered. Also, the parameter $\Lambda$ needs to be different. Can one separate the confinement and AF part and apply the same two parameters to hadron properties as well as to high density quark matter? Moreover, what happens to the strange star properties if the effect of temperature on gluon mass is considered?

Successful attempts have been made to construct a two parameter Richardson potential to explain energies and magnetic moments of baryons. Recently the magnetic moments of $\Delta^{++}$and $\Omega^{-}$with three $\mathrm{u}$ and three s valence quarks respectively have been accurately found in experiments. These sensitive properties are fitted with a modified Richardson potential with different scales for confinement $(\sim 350 \mathrm{MeV})$ and AF ( 100 MeV) (Bagchi et al. 2004). It was also shown that baryonic properties depend much on the confinement part and less on the AF part as baryons are confined quark systems. It is natural to apply this new potential to star calculations since they are constrained by baryonic data. Retaining the approach of Dey et al. (1998), we used this modified Richardson potential to derive our EOS.

\section{Modifications}

The properties of AF and confinement of quarks in the model is embedded in the potential we have used, i.e., the Richardson potential. We have extensively searched for the two parameter form of this potential that would separately reflect the properties of $\mathrm{AF}$ and quark confinement. In the light of the new modifications, the Richardson potential takes the form:

$$
\begin{aligned}
V_{i j}= & \frac{12 \pi}{27}\left[\frac{1}{\ln \left(1+\frac{\left(\boldsymbol{k}_{i}-\boldsymbol{k}_{j}\right)^{2}}{\Lambda^{2}}\right)}-\frac{\Lambda^{2}}{\left(\boldsymbol{k}_{i}-\boldsymbol{k}_{j}\right)^{2}}+\frac{\Lambda^{\prime 2}}{\left(\boldsymbol{k}_{i}-\boldsymbol{k}_{j}\right)^{2}}\right] \\
& \times \frac{1}{\left(\boldsymbol{k}_{i}-\boldsymbol{k}_{j}\right)^{2}}
\end{aligned}
$$

with $\Lambda^{\prime}$ the confinement property and $\Lambda$ asymptotic freedom.

The term $\left(\frac{1}{Q^{2} \ln \left(1+Q^{2} / \Lambda^{2}\right)}-\frac{\Lambda^{2}}{Q^{4}}\right)$ is asymptotically zero for large momentum transfer $Q^{2}=\left(\boldsymbol{k}_{i}-\boldsymbol{k}_{j}\right)^{2}$ and the term $\frac{\Lambda^{\prime 2}}{Q^{4}}$ explains the confinement reducing to a linear confinement for small $Q^{2}$.

Previously Bagchi et al. (2004) used the same form of the potential to fit baryonic properties and evaluated the appropriate values of $\Lambda$ and $\Lambda^{\prime}$. We have borrowed the same values $\left(\Lambda \sim 100 \mathrm{MeV}\right.$ and $\left.\Lambda^{\prime} \sim 350 \mathrm{MeV}\right)$ in our present calculation.

Ray et al. (2000b) calculated finite temperature effects on strange matter and strange stars, using the original one parameter Richardson potential. There, the temperature dependence of gluon mass was also not considered. We have incorporated this effect following Alexanian \& Nair (1995), who obtained a magnetic screening for the quark-gluon plasma at high density after performing self-consistence summation of one loop self-energy. It has the form $M \approx(2.384) \frac{C g^{2} T}{4 \pi}$ where $C=N$ for SU(N) gauge theory and $\mathrm{g}$ is the strong coupling constant $\frac{g^{2}}{4 \pi}=\alpha_{0}$. Adding this term to the usual density dependent one, the inverse screening length becomes:

$\left(D^{-1}\right)^{2}=\frac{2 \alpha_{0}}{\pi} \sum_{i=u, d, s,} k_{i}^{f} \sqrt{\left(k_{i}^{f}\right)^{2}+m_{i}^{2}}+7.152 \alpha_{0} T$

where we have used our standard notations as before. We have incorporated this form of the gluon mass (inverse screening length) for calculations of our new sets of SS EOSs.

\section{Results}

We check our new potential by calculating strange star properties at zero temperatures. We obtain different EOSs for different set of parameters tabulated in Table 1. For comparison, we also present one EOS SS1 obtained by Dey et al. (1998) using the unmodified potential in Table 2. In Fig. 1, we compared the $M-R$ plots for three of our new EOSs with SS1. The compactness $(M / R$ ratio) of the stars has increased for the new EOSs than compared to SS1. 


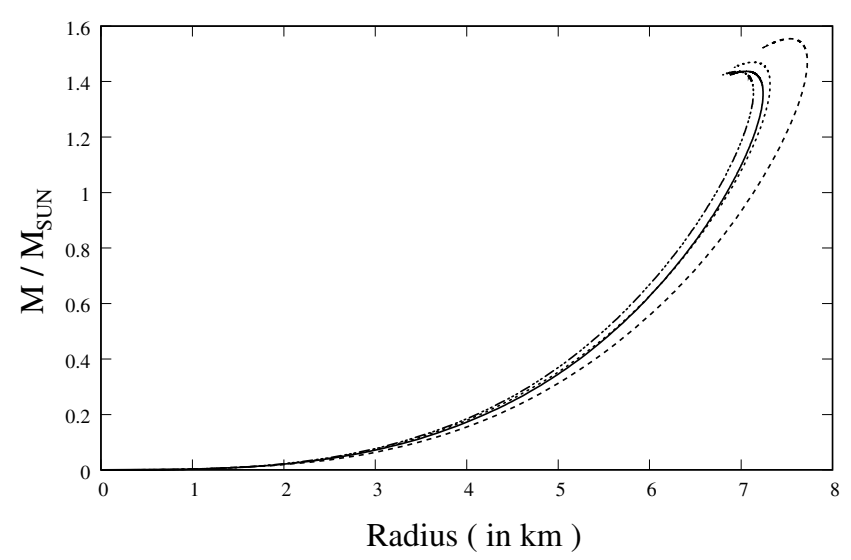

Fig. 1. Mass-radius relations for different parameter sets. Dashed curve is for EOS C, dotted curve is for EOS B, solid curve is for EOS SS1 and the dot-dashed curve is for EOS F.

From Tables 1 and 2, the nature of EOSs and $M-R$ relations do not change appreciably when using the modified potential if we adjust other model parameters suitably (see Fig. 1).

In our new EOSs, we have kept the value of the parameter $N$ to be the same (except EOS C, where we changed $N$ to see the effects). However, the value of the parameter $\alpha_{0}$ for our new EOSs is significantly different from SS1. Here, we chose a larger value because, as the confinement parameter has now increased from the previous value of $100 \mathrm{MeV}$ to $350 \mathrm{MeV}$, $\alpha_{0}$ should be increased to incorporate an earlier onset of deconfinement. The present value of $\alpha_{0}$ is $0.55-0.65$ compared to 0.20 of Dey et al. (1998). Such a value is in agreement with theoretical results (Ray et al. 2000a). From Table 1, we infer that the parameter $N$ has the greatest effect on the star structure and $M_{Q}$ the least.

With the parameters used to obtain EOS F, we have calculated strange star properties at finite temperatures. We have found that stable stellar structure can be obtained up to a temperature of $80 \mathrm{MeV}$ which is closer to Witten's conjectured temperature of cosmic separation of phases (100 MeV). Our results are shown in Figs. 2 and 3. The figures imply that in our strange star, the density $\left(\rho_{\mathrm{B}}\right)$ cannot be less than $4 \rho_{0}$ because negative pressure is physically not feasible. This means that the strange star has a sharp surface at a density $\rho_{\mathrm{B}} \sim 4 \rho_{0}$ where the pressure goes to zero. This property of the presence of sharp surfaces in strange stars has also been seen for other models, like the bag model, the chromo-dielectric model, etc.

In Fig. 4, we have shown both the density and temperature dependence of gluon mass. It is clear that at a given temperature, gluon mass increases with density and at a given density, gluon mass increases with temperature.

\section{Conclusions and summary}

A set of new EOSs for strange matter is presented - both for zero and very high $T$, using the Richardson potential with the value of $\Lambda^{\prime} \simeq 300$ to $350 \mathrm{MeV}$ and the value of $\Lambda=$ $100 \mathrm{MeV}$, the two scales for confinement and AF respectively. This explains both the properties of the deconfined quark
Table 1. Data for different EOSs, each having two different values for $\Lambda$ and $\Lambda^{\prime} ; \Lambda$ is always taken as $100 \mathrm{MeV}$. Change in the confinement scale $\Lambda^{\prime}$ does not change the minimum energy per baryon significantly.

\begin{tabular}{cccccccc}
\hline \hline $\begin{array}{c}\text { EOS } \\
\text { label }\end{array}$ & $\begin{array}{c}\Lambda^{\prime} \\
(\mathrm{MeV})\end{array}$ & $\begin{array}{c}M_{q} \\
(\mathrm{MeV})\end{array}$ & $N$ & $\alpha_{0}$ & $\begin{array}{c}(E / A)_{\min } \\
(\mathrm{MeV})\end{array}$ & $\begin{array}{c}M_{\max } \\
\left(M_{\odot}\right)\end{array}$ & $\begin{array}{c}R \\
(\mathrm{~km})\end{array}$ \\
\hline $\mathrm{A}$ & 350 & 325 & 3.0 & .55 & 874.0 & 1.532 & 7.411 \\
$\mathrm{~B}$ & 350 & 325 & 3.0 & .65 & 907.0 & 1.470 & 7.140 \\
$\mathrm{C}$ & 300 & 325 & 2.7 & .55 & 877.0 & 1.554 & 7.530 \\
$\mathrm{D}$ & 300 & 325 & 3.0 & .55 & 906.0 & 1.463 & 7.110 \\
$\mathrm{E}$ & 300 & 335 & 3.0 & .55 & 912.0 & 1.447 & 7.022 \\
$\mathrm{~F}$ & 350 & 345 & 3.0 & .65 & 920.3 & 1.436 & 6.974 \\
\hline
\end{tabular}

Table 2. One EOS (SS1) obtained by Dey et al. (1998) using an unmodified Richardson Potential with both $\Lambda$ and $\Lambda^{\prime}$ equal to $100 \mathrm{MeV}$.

\begin{tabular}{cccccccc}
\hline \hline $\begin{array}{c}\text { EOS } \\
\text { Label }\end{array}$ & $\begin{array}{c}\Lambda \\
(\mathrm{MeV})\end{array}$ & $\begin{array}{c}M_{q} \\
(\mathrm{MeV})\end{array}$ & $N$ & $\alpha_{0}$ & $\begin{array}{c}(E / A)_{\min } \\
(\mathrm{MeV})\end{array}$ & $\begin{array}{c}M_{\max } \\
\left(M_{\odot}\right)\end{array}$ & $\begin{array}{c}R \\
(\mathrm{~km})\end{array}$ \\
\hline SS1 & 100 & 310 & 3 & .20 & 889.0 & 1.437 & 7.06 \\
\hline
\end{tabular}

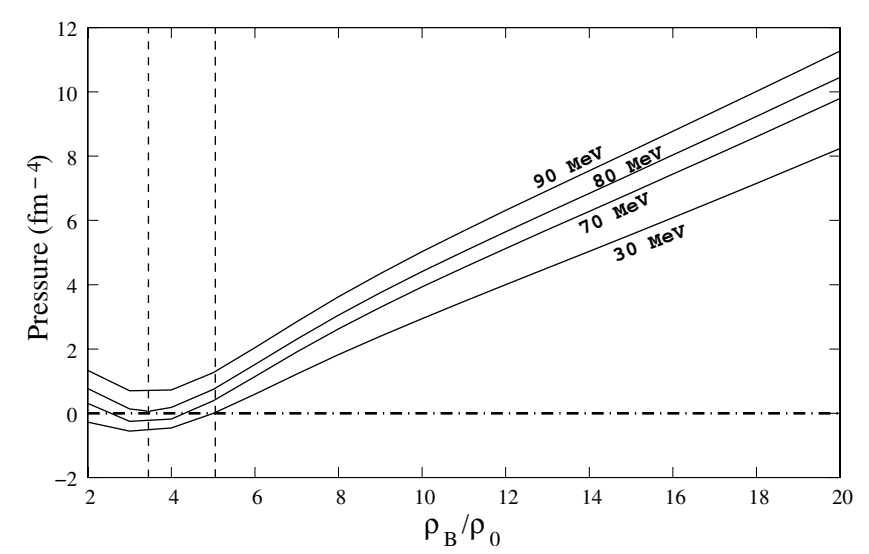

Fig. 2. EOSs at different temperatures, all with the same parameters as EOS F. The first vertical line from the left shows the location of the zero pressure (and hence stellar surface) for the EOS at $80 \mathrm{MeV}$, which is around 3.45. The second vertical line is for an EOS at $30 \mathrm{MeV}$, where the zero of the pressure is at 5.05 .

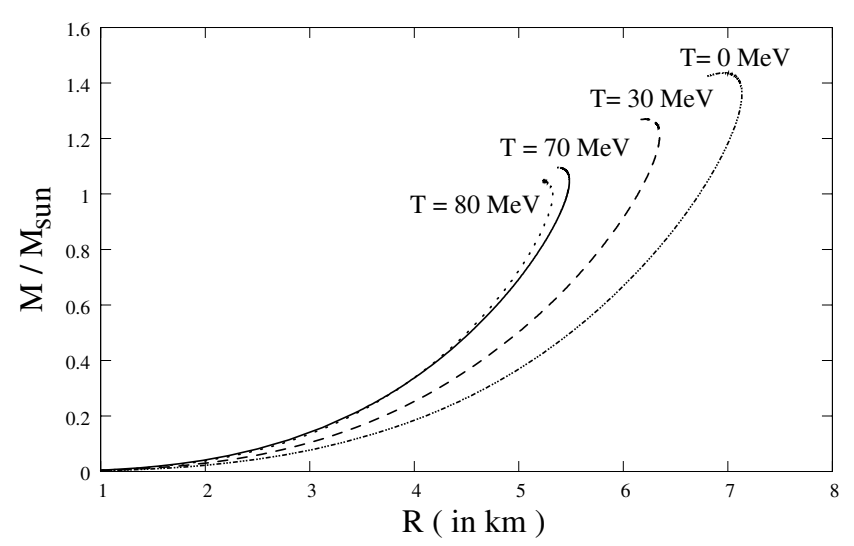

Fig. 3. Mass-radius relations at different temperatures, all with the same parameters as EOS F. 


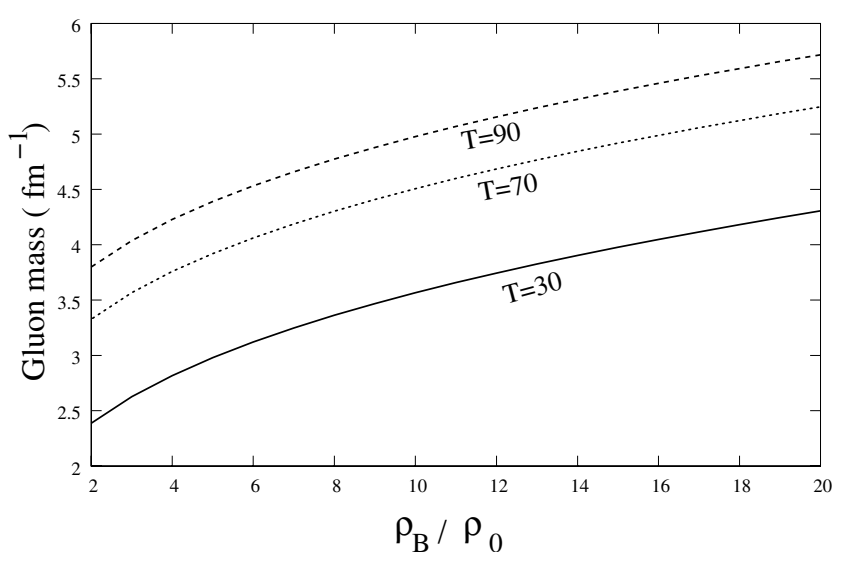

Fig. 4. Density and temperature dependence of gluon mass.

matter (our present work) and the properties of confined $3 \mathrm{u}$ and 3 s baryons (Bagchi et al. 2004). Although the confinement part $\Lambda^{\prime}$ is stronger, leading to a sharp surface, it is softened by medium effects, developing a screening length. Inside the star, the AF part is more important. We have found that for a wide range of parametric variation, the strange matter EOS gives a minimum of energy per baryon (E/A) that is lower than $(E / A)_{\mathrm{Fe}^{56}}=930.6 \mathrm{MeV}$ which ensures that the system is stable, and unlike neutron star - like structures, our strange stars are not solely bound by gravitational forces but also by strong forces. Moreover, considering temperature dependent screening of the potential, we have found that strange stars can sustain stable configurations up to a temperature of $80 \mathrm{MeV}$.

The indirect proof of the existence of strange stars is becoming more prominent because there are many observed features from compact objects that cannot be explained by the standard neutron star model, like spectral features and the controversies built around the star RX J1856.5-3754. Another feature, superbursts, faces difficulties in using the standard carbon burning scenario in the neutron star crust. The carbon, nitrogen and oxygen mass fraction $\left(Z_{\mathrm{CNO}}\right)$ must be larger than $Z_{\mathrm{CNO}, \odot \text {, }}$ where the latter refers to the standard value found in the sun (Cooper et al. 2005). Sinha et al. (2002) predicted that an alternative to the carbon burning scenario in a neutron star to explain superbursts, can be formation of diquark pairs on the strange star surface and their subsequent breaking, thus giving a continuous and prolonged emission of energy that can be comparable to the superbursts. Recently, Page \& Cumming (2005) tried to explain superbursts as carbon burning of matter accumulated on the electron cloud of a strange star. There are many other features that can be fitted better with a strange star than a neutron star model. So, the study of strange matter and strange stars is important and the formulation of a realistic strange star model is needed.
Acknowledgements. M.B., M.D. and J.D. acknowledge DST grant No. SP/S2/K-03/2001, Govt. of India and the IUCAA, Pune, India, for a fruitful visit.

\section{References}

Alcock, C., Farhi, E., \& Olinto, A. 1986, ApJ, 310, 261

Alcock, C., \& Olinto, A. 1989, Phys. Rev. D, 39, 1233

Alexanian, G., \& Nair, V. P. 1995, Phys. Lett. B, 352, 435

Bagchi, M., Dey, M., Daw, S., \& Dey, J. 2004, Nucl. Phys. A, 740, 109

Bagchi, M., Sinha, M., Dey, M., Dey, J., \& Bhowmick, S. 2005 [arXiv: astro-ph/0507609]

Blaschke, D., Grigorian, H., Poghosyan, D., Roberts, C. D., \& Schmidt, S. M. 1999, Phys. Lett. B, 450, 207

Boehm, C., Hooper, D., Silk, J., Casse, M., \& Paul, J. 2004, Phys. Rev. Lett., 92, 101301

Bodmer, A. R. 1971, Phys. Rev. D, 4, 1601

Bombaci, I., Parenti, I., \& Vidaña, I. 2004, ApJ, 614, 314

Burwitz, V., Haberl, F., Neuhäuser, R., et al. 2003, A\&A, 399, 1109

Cooper, R. L., Mukhopadhyay, B., Steeghs, D., \& Narayan, R. 2005, On the poduction and survival of carbon fuel for superbursts [arXiv:astro-ph/0508194v1]

Crater, H. W., \& van Alstine, P. 1984, Phys. Rev. Lett., 53, 1527

Dey, M., Bombaci, I., Dey, J., Ray, S., \& Samanta, B. C. 1998, Phys. Lett. B, 438, 123; Addendum 1999, 447, 352; Erratum 1999, 467, 303; 1999, Indian J. Phys. B, 73, 377

Dey, J., Dey, M., \& Le Tourneux, J. 1986, Phys. Rev. D, 34, 2104

Drake, J., Marshall, H. L., Dreizler, S., et al. 2002, ApJ, 572, 996

Fraga, E. S., Pisarski, R. D., \& Schaffner-Bielich, J. 2001, Phys. Rev. D, 63, 121702

Haensel, P., Zdunik, J. L., \& Schaeffer, R. 1986, A\&A, 160, 121

Kettner, C., Weber, F., Weigel, M. K., \& Glendenning, N. K. 1995, Phys. Rev. D, 51, 1440

Knodleseder, J., Lonjou, V., Jean, P., et al. 2003, A\&A, 411, L457

Lyne, A. G., Burgay, M., Kramer, M., et al. 2004, Science, 303, 1153

Malheiro, M., Fiolhais, M., \& Taurines, A. R. 2003, J. Phys. G, 29, 1045

Oaknin, D. H., \& Zhitnitsky, A. R. 2005, Phys. Rev. Lett., 94, 101301

Page, D., \& Cumming, A. 2005, Superbursts from strange stars [arXiv: astro-ph/0508444]

Rajagopal, K., \& Wilczek, F. 2000, [hep-ph/0011333]

Rajagopal, K., \& Wilczek, F. 2001, Phys. Rev. Lett., 86, 3492

Ray, S., Dey, J., \& Dey, M. 2000a, Mod. Phys. Lett. A, 15, 1301

Ray, S., Dey, J., Dey, M., Ray, K., \& Samanta, B. C. 2000b, A\&A, 364, 89

Ray, S., Dey, J., Dey, M., \& Bhowmick, S. 2004, MNRAS, 353, 825

Richardson, J. L. 1979, Phys. Lett. B, 82, 272

Sinha, M., Dey, M., Ray, S., \& Dey, J. 2002, MNRAS, 337, 1368

Sinha, M., Dey, J., Dey, M., Ray, S., \& Bhowmick, S. 2003, Mod. Phys. Lett. A, 18, 661

't Hooft, G. 1974, Nucl. Phys. B, 72, 461; 1974, Nucl. Phys. B, 75, 461

Weber, F. 2005, Progr. Part. Nucl. Phys., 54, 193

Witten, E. 1984, Phys. Rev. D, 30, 272

Witten, E. 1979, Nucl. Phys. B, 160, 57 